\title{
Simulated Annealing for Spin-glass-like Optimization Problems
}

\section{Morgenstern, Rüschlikon and Heidelberg}

(IBM Research Labs., and Ruprecht Karls Universität)

The technique of simulated annealing has arisen from the comparatively large research effort devoted over the past 15 years to the understanding of spin glasses. The technique has already had a strong impact on combinatorial optimization and its applications. As an example, in this article I describe the relations between spin-glass theory and experiments on the one hand, and the computer-aided chip design of electronic circuits on the other. In that context, simulated annealing was first introduced by S. Kirkpatrick et al. [1]. To understand the approach, let us first take a closer look at the area of physics where it was first developed.

\section{Spin-Glasses}

As some readers may recall [see Europhys. News 14 (1983) 12], spin glasses are disordered alloys with magnetic ions coupled by the so-called RKKY pair interaction, which exhibits damped oscillations as a function of the separation between two spins. Classic examples are $\mathrm{Gd}_{1-x} \mathrm{Mn}_{\mathrm{x}}$ and $\mathrm{Au}_{1-\mathrm{x}} \mathrm{Fe}_{\mathrm{x}}$. Such systems are described by the Hamiltonian

$$
-\beta \mathscr{H}=\sum_{<i, j>} J_{i j} S_{i} S_{j} .
$$

We concentrate here on short-range Ising-spin models $\left(S_{i}= \pm 1\right)$ with only nearest-neighbour couplings on either a square or a cubic lattice. Glass-like properties will arise if the interactions $J_{i j}$ are assumed randomly distributed with zero mean either between $\pm J$ or according to a Gaussian. For recent reviews, see Refs. 2-4.

A fundamental characteristic of spin glasses is their "frustration" or high ground-state degeneracy, usually accompanied by a large number of metastable states close in energy. These pro-

perties are illustrated by the (free-) energy "landscape" shown in Fig. 1, where $X$ denotes a representative coordinate in phase space, and $E$ the corresponding energy. Relatively high barriers separate different valleys. The bottoms of the valleys correspond to the above-mentioned ground states or metastable states. The key conjecture is that this picture also applies, at least qualitatively, to a wide variety of complex optimization problems in everyday life.

\section{NP-Complete Optimization Problems}

"NP-complete" describes a class of problems such that the computing time necessary to construct an optimal solution increases faster than any power of $N$ (the number of basic elements or spins); therefore they are called Non(deterministic) Polynomial. "Completeness" refers to problems of a given class that can be mapped onto each other in polynomial time. The search for a ground state in a spin glass belongs to the former class in the case of a three-dimensional lattice. The two-dimensional case only is P-complete. Other famous NP. complete problems are the "Travelling Salesman" and the "Placement and Wiring" of gates in chip design. As we shall see later, a huge variety of such problems exists in economics, biology, engineering, etc. In general, we can establish a connection between the "cost function" of an optimization problem and the total interaction energy of a spin-glass. In one obvious case, the cost function is the amount of money a travelling salesman has to spend to visit $N$ cities, one after the other. In placement and wiring problems, it is the wire length $L$ and/or the size of the chip.

Heuristic methods have already been

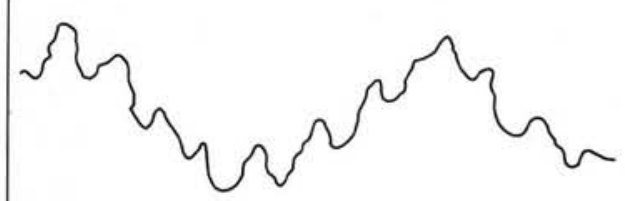

Fig. 1 - Schematic phase space cut through free energy landscape. developed for many such practical optimization problems. However, each such method is specially designed for a given problem and is therefore rather restricted in scope. In contrast, the "simulatedannealing" method provides a quite general approach. Annealing means controlled thermal treatment followed by slow cooling. For spin glasses, judicious "cooling" procedures have been developed to obtain ground-state configurations in computer simulations [2].

\section{Chip-Design for Physicists}

The placement or interconnexion problem for chips illustrates in a nutshell the simulated-annealing technique. Two-dimensional gate arrays are commercially available and consist of a certain fixed number of "cells" (for instance 2000) built from several transistors that perform a particular logical function. These cells are implemented on the gate array, and have to be wired by the user according to the logic of the chip which is specified in a "netlist". In view of the finite speed of electromagnetic signals, the chip performance depends critically on the total wire length $L$. Excessive congestion resulting in local overheating is a related problem to be avoided. Therefore, the total wire length is the cost function to be minimized. In view of the apparently large number of possibilities, this is done by the following Exchange Algorithm (see Fig. 2): Two cells are selected at random, and their positions exchanged. The difference in wire length $\Delta L$ is calculated. To avoid getting stuck prematurely in a metastable minimum, the disadvantageous configuration is not rejected automatically. Instead, it is assigned a probability $P$ and the exchange will be accepted if $P$ exceeds a number chosen randomly between $\mathrm{O}$ and 1 . The acceptance probability for the basic move is calculated according to the Monte-Carlo rule used to compute thermal equilibrium properties of interacting systems in classical statistical mechanics,

$$
\begin{array}{ll}
P=\exp (-\Delta L / T) & \text { for } \Delta L \geq 0 \\
P=1 & \text { otherwise, }
\end{array}
$$


Fig. 2 - Schematic twodimensional gate array. Logic cells (squares) are connected by wires. From Morgenstern I. and Würtz D., Z. Phys. B 6/7 (1987) 397.

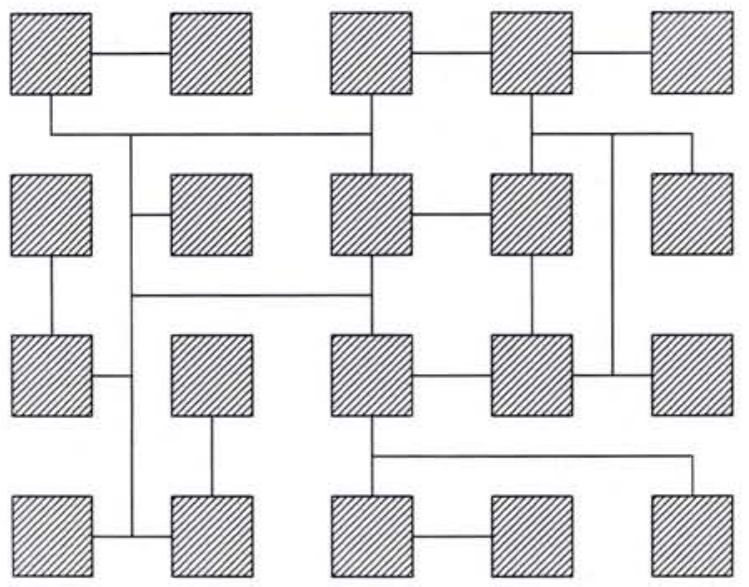

where $T$ is a fictitious temperature. After a large enough number of exchanges, the system "equilibrates" by spending most of the time in configurations with low values of $L$. To find one with minimum $L$, the system is "annealed" by slowly lowering $T$ in small increments. In spin-glass models, the analogous basic move is simply the flipping of a spin at a particular lattice site. The analogy to spin glasses can be fruitfully pursued. Thus, the emergence of a preferred wiring configuration is monitored by considering the mean square fluctuation of the wire length

$$
C=\left\langle L^{2}\right\rangle-\langle L\rangle^{2}
$$

as a function of $T$. It is natural to compare this quantity with its analogy in statistical mechanics which is proportioned to the specific heat. For the three-dimensional $\pm J$ spin glass, extensively investigated on a special-purpose computer at AT\&T Bell Laboratories, we notice a broad rounded peak on a linear temperature scale (Fig. 3a). A similar picture is obtained for the chip-design problem, but on a logarithmic scale, as seen in Fig. 3b. The corresponding annealing was performed according to the logarithmic cooling rule

$$
T_{\text {new }}=\alpha \cdot T_{\text {old }}
$$

where $\alpha=0.8-0.9$.

In the chip-design case, this may also produce a second peak denoted by "clustering" [1]. The simulation first singles out clusters of strongly connected cells that are absent in short-range spin-glass models. At lower temperature, "ordering" takes place, optimizing interconnexions inside the clusters. In Fig. 3a, the critical temperature $T_{c}$ for the three-dimensional spin glass is indicated. A finite critical temperature " $T_{c}$ " is also found in the finite gate-array problem, although $T_{\mathrm{c}}$ vanishes for the short-range spin glass in two dimensions. Below this temperature, the system is "frozen", i.e. the correlation length becomes comparable to the system size. "specific heat" for chip desing and spin glasses. The temperature dependence of this quantity can be computed relatively quickly, as it is dominated by frequent small-amplitude fluctuations. A rounded peak is a clear indication for "spin-glass-like" behaviour. Summarizing extensive experience, I recommend the following procedure:

(i) Plot the specific heat against a modified temperature scale which gives a spin-glass-like peak with comparable width.

(ii) Start annealing well above the peak. Take equidistant points on the new temperature scale.

(iii) Cool slowly between the peaks down to " $T_{c}$ " as outlined above. A conjecture by Grest et al. [5] deserves mention at this point. They looked at the cost-function value reached at the end of an annealing schedule as a function of the cooling rate. Studying mainly spinglass models, they obtained

$$
E(\tau) \sim 1 / \ell \mathrm{n} \tau
$$

where $\tau$ is the time spent in each temperature interval and $E$ is referred to its ground-state (optimum) value. This result is still hotly debated, especially in the spin-glass community [4]. Nonetheless, it or a modified scaling law would provide an answer to the most important question in simulated annealing. How much computing time must be spent to obtain a useful solution to an NP complete problem?

\section{Engineering Applications}

Simulated annealing has been used in a variety of problems. The strength of the method is certainly its generality. In principle, simulated annealing can be applied to all problems which can be attacked by iterative improvement [1]. Several such problems are described by Dreyfus et al. in Ref. 4, which presents a current overview of the situation. They include cloth cutting, time scheduling, planning of office buildings, and warehouse management.

More difficult tasks considered by the same authors include so-called "inverse red by exploiting the resemblance of the

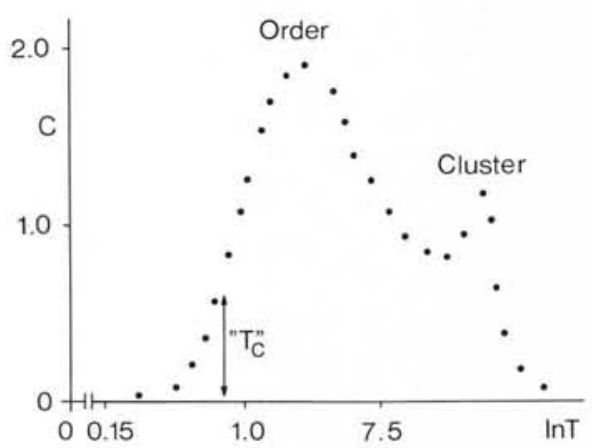

Fig. 3 - Computed specific heat of (a) three-dimensional spin-glass model (linear temperature scale), and (b) chip-design problem (logarithmic temperature scale). From Morgenstern I. and Würtz D., Z. Phys. B, to be published. (C) Springer-Verlag 1987. 
problems". An example is image processing where one tries to reconstruct the original and not just any acceptable optimal solution or image. One of the most spectacular applications is the interpretation of seismic data by Rothman [6]. He was able to reconstruct the geological structure of a terrain from information on the propagation of sound waves. Other impressive applications involve the reconstruction of three-dimensional objects from two-dimensional images in tomography, and the deblurring of binary images.

\section{Conclusion}

Simulated annealing offers a rather general approach to time-consuming problems. This is on the one hand its strength but on the other hand also its weakness. That means problem-oriented heuristic approaches may exist which are hard to beat. The general rule seems to be: the more money at stake in the solution of a particular problem, the better is the existing heuristics. This is certainly the case in chip design, where a general breakthrough for simulated an- nealing has not happened so far. But further research aims at this goal [4]. For a huge variety of less popular problems, simulated annealing is the way to proceed. In such a case, I encourage the reader to try my recipe. If the problem turns out to be "spin-glass-like", a comparatively good solution can be obtained for an optimal state. The future of simulated annealing certainly lies in the large variety of such problems waiting to be discovered in all branches of science, technology and economics.

\section{REFERENCES}

1. Kirkpatrick S., Gelatt C.D. and Vecchi M.P., Science 220 (1983) 671.

2. "Heidelberg Colloquium on Spin-Glasses", in Lecture Notes in Physics 192, eds. J.L. van Hemmen and I. Morgenstern (Springer-Verlag, Heidelberg) 1983.

3. Binder K. and Young A.P., Rev. Mod. Phys. 58 (1986) 801.

4. "Heidelberg Colloquium on Glassy Dynamics", in Lecture Notes in Physics 275, eds. J.L. van Hemmen and I. Morgenstern (Springer-Verlag, Heidelberg) 1987.

5. Grest G.S., Soukoulis C.M. and Levin K., Phys. Rev. Lett. 56 (1986) 1148.

6. Rothman D., Geophysics 50 (1985) 2784.

\section{SOUIDS}

Continuing the series of Workshops especially conceived to interest our Associate Members, will be one on the "SOUID: State of the Art, Perspectives and Applications". It will be held at the CNR headquarters in Rome from 22-24 June 1988 . Over $2 \frac{1}{2}$ days the Workshop will be discussing current developments and will also consider the impact of the new oxide superconductors.

Organising the Workshop are Profs. P. Carelli of the University of Aquila and A. Paoletti of the CNR together with the EPS Secretariat. A maximum number of 50 participants is envisaged including nine review speakers. Dead-line for returning Pre-registration forms (available at the Secretariat) is 29 February 1988. Invitations will be sent out in April. No proceedings are planned, but some papers will be available at the time.

Staff members of EPS Associate Members and IOMs will be charged Sw.Fr. 200.-; all others Sw.Fr. 400.-, although invited speakers from Universities may apply for a reduction.

\section{CATEGORY 4a)}

M. Al-Bakir, Baghdad, Iraq

E. Canessa, Norwich, UK

Ch. Chang, Berlin W.

D.G. Gratias, Vitry, F

T.T. Luong, Caen, F

\section{CATEGORY 4c)}

Danish Physical Society

K. Dyrbye, Aarhus

J. Mygind, Longby

I. Shim, Ballerup

Eötvös Lorand Physical Society

L. Koltai, Budapest

I. Vomberg, Budapest

Finnish Physical Society

S.S.H. Kasi, Kerava

\section{New Members of the European Physical Society}

German Physical Society

Walter Buckel, Karlsruhe

R. Kalla, Münster

S. Martin, Murray Hill, NJ, USA

W.F. Staudt, Kaiserslautern

T.A. Vilgis, Mainz

W.F. Wreszinski, Sao Paulo, Brazil

Hellenic Physical Society

G. Brodimas, Patras

\section{The Institute of Physics}

C.M.C. de Castilho,

Salvador-Bahia, Brazil

D. Evans, Durham

P.D. Greaves, Copenhagen, DK

S. Holloway, Liverpool

A. Öztarhan, Izmir, TR
Italian Physical Society

U. Amaldi, Geneva, $\mathrm{CH}$

The Netherlands' Physical Society E. de Boer, Nijmegen M.T. Rekveldt, Delft

Physical Section, Union of Czechoslovak Mathematicians and Physicists

J. Mrnustik, Boskovice

Polish Physical Society

J. Kasperczyk, Czestochowa

J.K. Moscicki, Krakow

Romanian National Committee for Physics

G. Constantinescu, Bucharest
Spanish Royal Society of Physics

J. Bausells, Barcelona

A. Criado, Sevilla

J. Marro, Granada

P. Wahnon, Madrid

\section{Swedish Physical Society}

O. Gustavsson, Haninge

Swiss Physical Society

W. Joss, Varces, F

P. Muralt, Balzers, FL

\section{CATEGORY 4d)}

American Physical Society

D.S. Barton, Upton, NY

G. Margaritondo, Stoughton, WI

H. Morawitz, San Jose, CA

Australian Institute of Physics

A.B. Murphy, Garching, D
EPS Divisions, Sections and Group Astronomy and Astrophysics Division Solar Physics Section Atomic and Molecular Physics Division Atomic Spectroscopy Section. Chemical Physics Electronic and Atomic Collisions Molecular Physics Computational Physics Group Condensed Matter Division Liquids Section Low Temperature Physics Section Macromolecular Physics Magnetism Magnetism Metal Physics Semiconductors and insula
Surfaces and Interfaces Surfaces and Interfaces
High Energy \& Particle Physics Division Interdiv, Group on Exptl. Phys. Control Systems Interdiv. Group on Physics for Developmen Nuclear Physics Division Optics Division Plasma Physics Division Quantum Electronics Division
Europhysics News is the official journal of the European Physical Society which comprises 29 National Societies, Academies and Group, about 4000 Individual Members and 75 Associate Members. Governing bodies of EPS are the General Meeting, Council and an elected Executive Committee responsible for detailed policy. EPS promotes the collaboration of physicists policy. EPS promotes the collaboration of physicists throughout Europe, organising and harmonising conferences and publications, improving physics education, ships to sponsored schools in Erice. EPS publishes in addition to Europhys. News, Europhysics Letters (in partnership with national societies), European Journal of Physics (in partnership with The UK Inst. of Phys.). and European Conference Abstracts. Individual Members receive Europhys. News free of charge (price to insts.: Sw.Fr. 90. - /a), Europhys. Lett. at Sw.Fr. 70.-la (insts. 595.-), rebates on many other publications and on conference fees. Annual EPS membership fee for Individual Members belonging to an EPS member society is: Sw.Fr. 44.-; independent members: Sw.Fr. 132.-; members of a Collaborating Society: Sw.Fr. 55.-

\author{
Editor: E.N. Shaw \\ Editorial Board: \\ A. Baratoff, F. James, M. Lehmann, \\ M. Mayor, J. Muller, M. Siegrist \\ Editorial and Advertising Office at the EPS \\ Secretariat \\ Address: EUROPEAN PHYSICAL SOCIETY \\ P.O. Box 69 , \\ CH-1213 Petit-Lancy 2 \\ Switzerland \\ Telephone: Geneva (22) 931130 \\ Telex: 428024 eps ch \\ Cables: europhys genève \\ Printed by: Pfirter frères se \\ $\mathrm{CH}-1213$ Petit-Lancy/Switzerland
}

